\title{
Fructose Metabolism and Cardiac Metabolic Stress
}

\author{
M. Annandale ${ }^{1}$, L. J. Daniels ${ }^{1,2}$, X. Li ${ }^{1}$, J. P. H. Neale ${ }^{1}$, A. H. L. Chau ${ }^{3}$, H. A. Ambalawanar ${ }^{1}$, \\ S. L. James ${ }^{1}$, P. Koutsifeli ${ }^{1}$, L. M. D. Delbridge ${ }^{3}$ and K. M. Mellor ${ }^{1,3 *}$ \\ ${ }^{1}$ Department of Physiology, Faculty of Medical and Health Sciences, University of Auckland, Auckland, New Zealand, ${ }^{2}$ Oxford \\ Centre for Diabetes, Endocrinology and Metabolism, Radcliffe Department of Medicine, University of Oxford, Oxford, \\ United Kingdom, ${ }^{3}$ Department of Physiology, School of Biomedical Sciences, University of Melbourne, Melbourne, VIC, Australia
}

\section{OPEN ACCESS}

Edited by:

Arpeeta Sharma,

Baker Heart and Diabetes Institute, Australia

Reviewed by: Fabio Vivarelli, University of Bologna, Italy Dongze Qin,

Albert Einstein College of Medicine, United States

*Correspondence: K. M. Mellor k.mellor@auckland.ac.nz

Specialty section: This article was submitted to Translational Pharmacology, a section of the journal

Frontiers in Pharmacology

Received: 15 April 2021 Accepted: 07 June 2021 Published: 29 June 2021

Citation: Annandale M, Daniels LJ, Li X Neale J PH, Chau AHL, Ambalawanar HA, James SL, Koutsifeli $P$, Delbridge $L M D$ and Mellor KM (2021) Fructose Metabolism and Cardiac Metabolic Stress. Front. Pharmacol. 12:695486. doi: 10.3389/fphar.2021.695486
Cardiovascular disease is one of the leading causes of mortality in diabetes. High fructose consumption has been linked with the development of diabetes and cardiovascular disease. Serum and cardiac tissue fructose levels are elevated in diabetic patients, and cardiac production of fructose via the intracellular polyol pathway is upregulated. The question of whether direct myocardial fructose exposure and upregulated fructose metabolism have potential to induce cardiac fructose toxicity in metabolic stress settings arises. Unlike tightly-regulated glucose metabolism, fructose bypasses the rate-limiting glycolytic enzyme, phosphofructokinase, and proceeds through glycolysis in an unregulated manner. In vivo rodent studies have shown that high dietary fructose induces cardiac metabolic stress and functional disturbance. In vitro, studies have demonstrated that cardiomyocytes cultured in high fructose exhibit lipid accumulation, inflammation, hypertrophy and low viability. Intracellular fructose mediates posttranslational modification of proteins, and this activity provides an important mechanistic pathway for fructose-related cardiomyocyte signaling and functional effect. Additionally, fructose has been shown to provide a fuel source for the stressed myocardium. Elucidating the mechanisms of fructose toxicity in the heart may have important implications for understanding cardiac pathology in metabolic stress settings.

Keywords: carbohydrate metabolism, diabetic cardiomyopathy (DCM), fructolysis, cardiac lipogenesis, advanced glycation end - products

\section{INTRODUCTION}

The prevalence of insulin resistance and type 2 diabetes has increased considerably in the last few decades (Ford et al., 2002). It is estimated that currently more than 425 million people globally have diabetes, with numbers projected to increase to $\sim 700$ million by 2045 (Cho et al., 2018). Diabetic patients have an increased risk of developing cardiovascular disease and heart failure (Nichols et al., 2001; Lind et al., 2014; Tancredi et al., 2015). Diabetic cardiomyopathy constitutes a distinct cardiac pathology, independent from other cardiovascular disorders (Huynh et al., 2014; Delbridge et al., 2016; Ritchie and Abel, 2020). Population studies have demonstrated that excess fructose consumption is linked with the development of diabetes and cardiovascular disease (Malik et al., 2010; Johnson et al., 2013; DiNicolantonio et al., 2015; Stanhope, 2016). There is some controversy about whether this association is due to increased energy consumption, or partially mediated by the unique pathological effects of fructose on organ systems (Stanhope, 2016). In pre-clinical rodent studies, isocaloric high dietary fructose induces cardiac metabolic stress and functional disturbance (Mellor et al., 2010b; Mellor et al., 2011b, Mellor et al., 2012). High dietary fructose has also been 
shown to induce early signs of diastolic dysfunction, alter expression of mitochondrial, apoptotic and oxidative stressrelated proteins, and lead to accumulation of cardiac lipid species (Axelsen et al., 2010; Szúcs et al., 2019). Clinically, both circulating and cardiac fructose levels are elevated in diabetic patients (Kawasaki et al., 2002; Daniels et al., 2021), suggesting that direct myocardial exposure to fructose is heightened in a diabetic setting. Pathological outcomes of cardiomyocyte fructose exposure have been demonstrated in tightly controlled in vitro experimental settings (Zhang et al., 2016a; Zhao et al., 2017; Fan et al., 2018) and evidence is emerging to support a case for fructose as a pathological agent in the heart. Under normal conditions fatty acids are the predominant cardiac metabolic substrate for ATP supply, and the heart displays remarkable metabolic flexibility in using other substrates such as carbohydrates, amino acids and ketones in response to metabolic stress (Kolwicz et al., 2013). In vitro studies suggest that cardiomyocytes have the capacity to metabolize fructose (Mellor et al., 2011a) but the extent to which the heart utilizes fructose as an energy substrate in physiological or pathological settings has not been established. This review aims to survey the clinical and experimental literature assessing fructose involvement in cardiac energy management, cardiomyocyte signaling and metabolism. The potential molecular mechanisms underlying a role for increased fructose exposure in the development of cardiac metabolic stress are examined.

\section{SOURCES OF CARDIAC FRUCTOSE}

Fructose is a naturally occurring ketohexose sugar (i.e., containing a ketone and six carbons) that is commonly found in fruits or vegetables, where it can either exist alone in its free form or in combination with glucose in the form of sucrose (Rumessen, 1992). Additionally, sweeteners such as high fructose corn syrup have contributed to increased fructose consumption (Pereira et al., 2017). In the heart, cellular fructose content may be determined by the extent of fructose uptake from the systemic circulation and/or by intracellular fructose production via polyol (sorbitol) pathway flux. Tissue fructose levels are typically measured using enzymatic assay or mass-spectrometry based approaches (Kashiwagi et al., 1992; Daniels et al., 2021). Our recent study provided the first evidence that cardiac tissue fructose and sorbitol levels are significantly elevated in type 2 diabetic patients (Daniels et al., 2021). Similarly, cardiac fructose levels have been reported to be elevated in experimental rodent models of both type 1 and type 2 diabetes (Kashiwagi et al., 1992; Lal et al., 1997; Li et al., 2008). These findings suggest that elevated cardiac fructose content and metabolism may be an important characteristic of diabetic cardiomyopathy.

\section{Extracellular Sources of Cardiac Fructose}

Increased circulating fructose has been reported in diabetes, with diabetic patients exhibiting $50-80 \%$ increased serum fructose concentration relative to healthy subjects (Kawasaki et al., 2002; Gul et al., 2009). Dietary fructose is absorbed across the intestinal brush border via the facilitative glucose transporter
(GLUT)-5 (Burant et al., 1992; Drozdowski and Thomson, 2006). Low level supply of dietary fructose $(<0.2 \mathrm{~g} / \mathrm{kg})$ is almost completely $(\sim 90 \%)$ metabolized into glucose, lactate and glycerate in intestinal cells (Jang et al., 2018). High level dietary fructose $(\geq 1 \mathrm{~g} / \mathrm{kg})$ saturates intestinal clearance resulting in spillover into the portal vein via basolateral GLUT2 transporters (Leturque et al., 2005; Douard and Ferraris, 2008; Jang et al., 2018). Portal vein fructose is then taken up by hepatocytes via GLUT2 and GLUT5, where it is predominantly metabolized into glucose and lactate in the liver (Sun and Empie, 2012). However human isotope-labeling studies have demonstrated that a significant proportion of ingested fructose escapes splanchnic extraction ( $15 \%$ of a $30 \mathrm{~g}$ fructose load) and enters the systemic circulation (Francey et al., 2019). Several fructose transporters such as GLUT5, GLUT11, and GLUT12 have been detected in tissues such as the heart, skeletal muscle, brain, adipose tissue and others (Mellor et al., 2010a). Reported values of fructose levels in the systemic circulation vary widely, likely due to differences in methodologies used to measure fructose, with enzymatic assays often generating higher values than mass spectrometrybased approaches. Clinical guidelines have not been established for defining the "normal" range of human blood fructose levels and literature values for healthy subjects vary widely. Human fasted serum fructose levels are typically reported between 5 and 400 M (Kruszynska et al., 1993; Tran et al., 2010; Le et al., 2012) and values as high as $1900 \mu \mathrm{M}$ have been reported in healthy subjects (Hui et al., 2009). Clearance of fructose by the small intestine and the liver appears to maintain baseline fructose concentrations predominantly at the sub-millimolar level, but post-prandial serum fructose levels have been shown to increase into the millimolar range following acute dietary interventions (Kruszynska et al., 1993; Hui et al., 2009; Low et al., 2018). Dietary-derived fructose may therefore play a significant role in determining fructose spillover into the systemic circulation leading to significant cardiac fructose exposure and potential cardiomyocyte fructose uptake, particularly in the context of diabetes.

The mechanisms and kinetics of cardiomyocyte fructose uptake are not fully elucidated. Cardiac expression of fructose transporters, GLUT5, GLUT11 and GLUT12 has been reported (Mellor et al., 2010a, Mellor et al., 2011a; Daniels et al., 2021). GLUT11 and GLUT12 transport glucose and fructose in a competitive manner. Given that circulating millimolar glucose levels exceed reported fructose levels in the micromolar range, GLUT11 and GLUT12 are unlikely to play a significant functional role in cardiomyocyte fructose uptake (Rogers et al., 2002, Rogers et al., 2003; Scheepers et al., 2005). Additionally, the lack of mouse orthologue for GLUT11 has limited investigations using genetic manipulation tools. In contrast, the GLUT5 transporter is highly specific for fructose with a low affinity for glucose transport (Kayano et al., 1990; Burant et al., 1992). Thus, GLUT5-mediated fructose transport is likely to occur even in the context of relatively high circulating glucose concentrations. Whether fluctuations in blood glucose levels influence cardiomyocyte fructose uptake and metabolism, has not been investigated. Emerging evidence suggests that cardiac GLUT5 expression is 


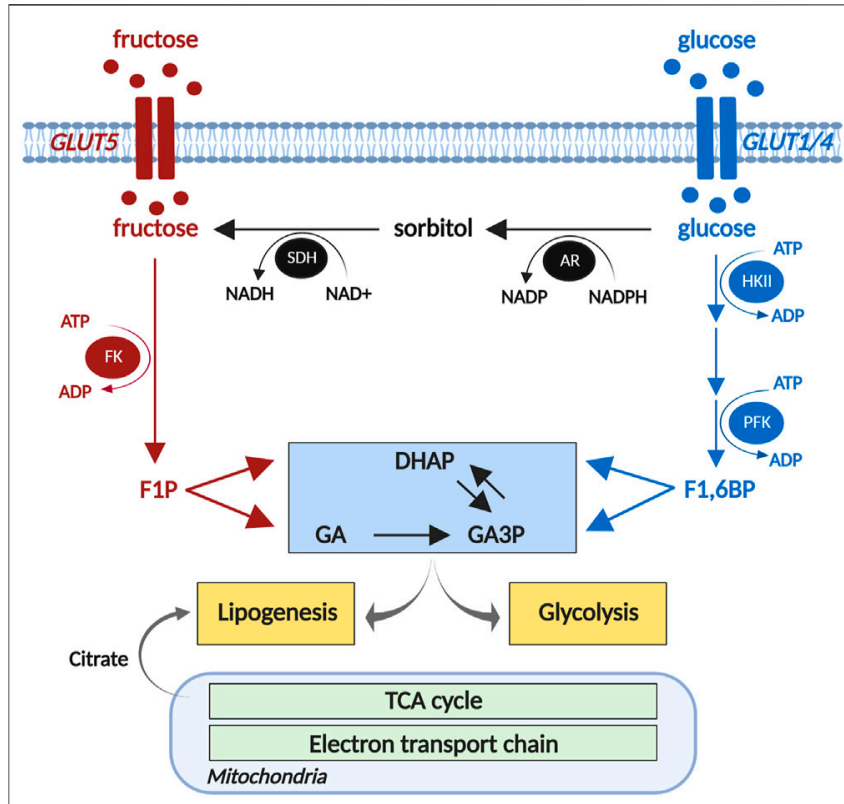

FIGURE 1 | Cardiomyocyte fructose metabolism. Fructose is phosphorylated by fructokinase (FK) to fructose 1-phosphate (F1P), and metabolized to triose phosphates, dihydroxyacetone phosphate (DHAP) and glyceraldehyde (GA). The polyol pathway (black) converts glucose to sorbitol via aldose reductase (AR), and sorbitol to glucose via sorbitol dehydrogenase (SDH). DHAP and GA are converted to glyceraldehyde 3phosphates (GA3P) and provide substrates for glycolysis to produce acetylCoA to enter the mitochondrial TCA cycle. DHAP and TCA cycle-derived citrate contribute to lipid biosynthesis. HKII, hexokinase 2; PFK, phosphofructokinase; TCA, tricarboxylic acid. Created using biorender.com. Adapted from Daniels et al., 2021.

upregulated in disease states, demonstrated by increased GLUT5 mRNA expression in type 2 diabetic Zucker diabetic fatty rat cardiac tissue (Daniels et al., 2021), and in left-ventricular biopsies from aortic stenosis and hypertrophic cardiomyopathy patients (Mirtschink et al., 2015). In non-cardiac cells, fructose exposure has been shown to increase GLUT5 expression (David et al., 1995; Jiang et al., 2001; Patel et al., 2015) indicating that GLUT5 can be positively regulated by its own substrate. Upregulated fructose uptake in the diabetic heart may contribute to increased intracellular cardiomyocyte fructose, and further investigation is warranted.

\section{Intracellular Sources of Cardiac Fructose}

An alternative (non-dietary) source of cardiac fructose is derived from endogenous fructose production via the polyol pathway. In this process, glucose is metabolized into sorbitol via aldose reductase, which is then converted to fructose by sorbitol dehydrogenase (Figure 1; Tang et al., 2012). Cardiac capacity to convert glucose to fructose has been demonstrated in ex vivo rat hearts, where $2 \mathrm{~h}$ of high glucose $(33 \mathrm{mM})$ perfusion significantly increased cardiac fructose levels (Tang et al., 2010). We have recently shown that cardiac sorbitol levels are increased in human type 2 diabetic hearts (Daniels et al., 2021), consistent with similar findings in type 1 and type 2 diabetic rodent models (Kashiwagi et al., 1992; Lal et al., 1997; Li et al.,
2008). Variable findings have been reported regarding the expression of aldose reductase in diabetic hearts. Increased aldose reductase expression is evident in spontaneous type 2 diabetic BBZ rat hearts at 48 weeks of age (Li et al., 2008), contrasting with decreased aldose reductase expression in type 1 diabetic mouse hearts at 3 weeks post-streptozotocin administration (Iwata et al., 2007). These differences may in part be due to the diabetic models used and duration of diabetes. Despite the disparities evident in protein expression, aldose reductase enzyme activity appears to be consistently elevated in type 1 diabetic rodent hearts (Iwata et al., 2007; Sharavana et al., 2017). Sorbitol dehydrogenase protein expression is increased in spontaneous type 2 diabetic BBZ rat hearts at 48 weeks of age ( $\mathrm{Li}$ et al., 2008), and increased enzyme activity has been reported in type 1 diabetic (streptozotocin-induced) rat hearts (Sharavana et al., 2017). These studies suggest that cardiac fructose production via the polyol pathway may be upregulated in diabetic settings. This finding appears paradoxical in the context of impaired cardiomyocyte glucose uptake and availability due to insulin resistance in type 2 diabetes or insulin deficiency in type 1 diabetes (Desrois et al., 2004; van den Brom et al., 2009). Interestingly, cardiac sorbitol content is correlated with diastolic dysfunction in type 2 diabetic patients (Daniels et al., 2021), suggesting that elevated polyol pathway flux may present an early risk factor for the progression of diabetic cardiomyopathy. High glucose-induced cardiac contractile dysfunction and oxidative stress were ameliorated by inhibition of the polyol pathway enzymes, aldose reductase and sorbitol dehydrogenase (Tang et al., 2010). There is some indication that systemic inhibition of the polyol pathway has favorable cardiac outcomes in vivo, with oral administration of an aldose reductase inhibitor (Zopolrestat) leading to improvement in left ventricular ejection fraction in diabetic patients over 1 year of treatment (Johnson et al., 2004). Whether these cardiac effects are secondary to changes in other tissues is yet to be determined.

\section{FRUCTOSE METABOLISM IN THE HEART}

The capacity of the heart to metabolize fructose was first observed using isolated adult rat cardiomyocytes, where fructose, in the absence of glucose, sustained cardiomyocyte excitationcontraction coupling acutely (Mellor et al., 2011a). In cultured neonatal rat ventricular myocytes, $1 \mathrm{mM}$ fructose exposure for $24 \mathrm{~h}$ significantly increased glycolytic capacity (Daniels et al., 2021). Cellular fructose metabolism is initiated by phosphorylation of free fructose by fructokinase (also known as ketohexokinase) to form fructose 1-phosphate (Figure 1). Fructose 1-phosphate is metabolized to dihydroxyacetone phosphate (DHAP) and glyceraldehyde (GA) which can enter the glycolytic pathway payoff phase (Khitan and Kim, 2013; Mirtschink and Krek, 2016). Unlike tightly regulated glucose metabolism, fructose bypasses the rate-limiting glycolytic enzyme, phosphofructokinase (Khitan and Kim, 2013), and proceeds through glycolysis in an unregulated manner. Experimental upregulation of fructokinase (via genetic splicing) increased fructose metabolic flux in cultured neonatal 
mouse cardiomyocytes, as evidenced by increased fructosederived fructose 1-phosphate (Mirtschink et al., 2015). Increased fructokinase expression is evident in Zucker type 2 diabetic rat hearts (Daniels et al., 2021) and in ventricular biopsies of aortic stenosis or hypertrophic cardiomyopathy patients (Mirtschink et al., 2015). Collectively these studies provide evidence that cardiomyocytes have the capacity to upregulate fructose metabolism in both diabetic and heart failure settings.

Fructose-derived glycolytic metabolism generates pyruvate, a well-known substrate of the tricarboxylic acid cycle (TCA) in the mitochondria. Thus it is likely that disturbances in fructose metabolism may have downstream impact on oxidative metabolism. Impaired cardiac mitochondrial function is a common feature of diabetic cardiomyopathy (Diamant et al., 2003; Boudina et al., 2005; Anderson et al., 2009; Pham et al., 2014), but no studies to date have directly investigated whether diabetic mitochondrial dysfunction is linked to cardiac fructose metabolism in vivo. In vitro evidence suggests that increased fructose exposure induces alterations in cardiomyocyte mitochondrial metabolism. Neonatal rat ventricular myocytes cultured in $25 \mathrm{mM}$ fructose for $48 \mathrm{~h}$ exhibit increased mitochondrial complex I and II hydrogen peroxide production (Zhang et al., 2016b), consistent with inefficient mitochondrial electron transport chain function. In H9c2 cardiomyoblasts, $50 \mathrm{mM}$ fructose exposure for $24 \mathrm{~h}$ significantly decreased mitochondrial respiration, and ATP production, and increased proton leak (Park et al., 2018). In contrast, mitochondrial function was unchanged in cultured neonatal rat ventricular myocytes exposed to physiological levels $(1 \mathrm{mM})$ of fructose for $24 \mathrm{~h}$ (Daniels et al., 2021), suggesting that fructose influence on cardiomyocyte mitochondrial function may only be evident at supra-physiological fructose concentrations. In a setting of enhanced fructose metabolism induced by fructokinase-C overexpression, relatively low-level fructose exposure $(25 \mu \mathrm{M}$ fructose) suppressed mitochondrial oxidative phosphorylation in neonatal mouse cardiomyocytes (Mirtschink et al., 2015). This finding suggests that in a setting of upregulated fructose metabolism, even low levels of fructose may be detrimental to cardiomyocyte mitochondrial function.

\section{PATHOLOGICAL OUTCOMES OF CARDIOMYOCYTE FRUCTOSE EXPOSURE}

\section{Fructose-Induced Cardiomyocyte Lipid Accumulation}

Lipid accumulation is a key feature of the diabetic heart (Regan et al., 1977) and is considered to be pathologic. Experimentally, a high fructose diet increases cardiac triacylglycerol (TAG) synthesis and induces cardiac TAG accumulation (Faeh et al., 2005; Stanhope et al., 2009; Fan et al., 2018). Fructose has the potential to promote cardiomyocyte lipid production either as a substrate or via upregulation of enzymes involved in de novo lipogenesis (Mirtschink et al., 2015; Fan et al., 2018; Lalowski et al., 2018). Incubating primary mouse cardiomyocytes in $\left[{ }^{3} \mathrm{H}\right]-$ labeled fructose overnight led to direct incorporation of fructosederived $\left[{ }^{3} \mathrm{H}\right]$ in lipids (Mirtschink et al., 2015), supporting the contention that fructose is a lipid substrate in the heart. Two pathways of fructose metabolism may lead to de novo lipogenesis. Citrate produced from fructose-derived DHAP metabolized through glycolysis and subsequent TCA cycle, may be metabolized to fatty acid-CoA (Saponaro et al., 2015). Alternatively, fructose-derived DHAP may be converted into glycerol 3-phosphate via glyceroneogenesis. Both fatty acidCoA and glycerol 3-phosphate are substrates for de novo TAG synthesis (Saponaro et al., 2015). Accumulation of lipid droplets is evident in primary rat cardiomyocytes and $\mathrm{H} 9 \mathrm{c} 2$ cardiomyoblasts following $1 \mathrm{mM}$ fructose exposure for $24 \mathrm{~h}$ (Daniels et al., 2021). Similarly, fructose exposure increased TAG content in $\mathrm{H} 9 \mathrm{c} 2$ cardiomyoblasts and primary mouse cardiomyocytes (Kang et al., 2016; Fan et al., 2018). Fructoseinduced cardiomyocyte lipid accumulation may in part be due to changes in gene expression, as primary mouse cardiomyocytes cultured in $5 \mathrm{mM}$ fructose for $24 \mathrm{~h}$ exhibit increased mRNA expression of enzymes involved in de novo production of fatty acid-CoA, FAS and stearoyl-CoA desaturase-1 (SCD1) (Fan et al., 2018). Upregulated fructose metabolism has also been linked to cardiomyocyte lipid production, as fructokinase-C overexpression significantly increased lipid content in neonatal mouse cardiomyocytes (Mirtschink et al., 2015). Given the evidence that cardiac fructose is elevated in diabetic hearts in vivo and fructose exposure in vitro promotes cardiomyocyte lipid accumulation, a case can be made that fructose may contribute to lipid accumulation in diabetic cardiomyopathy, but to date this has not been directly investigated.

\section{Fructose-Derived Cardiomyocyte Post-translational Modifications}

Intracellular cardiac proteins can be altered through irreversible non-enzymatic glycation modification of amino acid residues known as advanced glycation end products (AGEs). The AGE formation process is initiated by the attachment of a hexose sugar (e.g. glucose, fructose) to a protein to form a Schiff base. Schiff bases are rearranged into AGE intermediates (e.g., Amadori products) and ultimately form AGEs following a series of oxidation-based Maillard reactions (Cho et al., 2007). Production of AGEs and their interaction with receptors for AGEs (RAGEs) can contribute to contractile dysfunction, increased inflammation and oxidative stress in cardiomyocytes (Bodiga et al., 2014). The molecular mechanisms underlying the link between AGE formation and diabetic cardiac pathology are not fully elucidated but there is some indication that direct obstruction of sarcomeric proteins is involved (Papadaki et al., 2018). Clinically, increased serum AGEs are associated with impaired left ventricular diastolic function in diabetic patients (Berg et al., 1999; Sveen et al., 2014). Experimental rodent models of diabetes exhibit increased AGE modification of cardiac proteins involved in excitation-contraction coupling (Bidasee et al., 2003, 2004). Interestingly, formation of fructose-derived AGEs occurs much more rapidly than glucose-derived AGEs (McPherson et al., 1988; Suárez et al., 1989), suggesting that fructose poses a greater risk for protein modification than glucose. The open-chain conformation of fructose likely results 
TABLE 1 | Fructose-induced cardiomyocyte pathology.

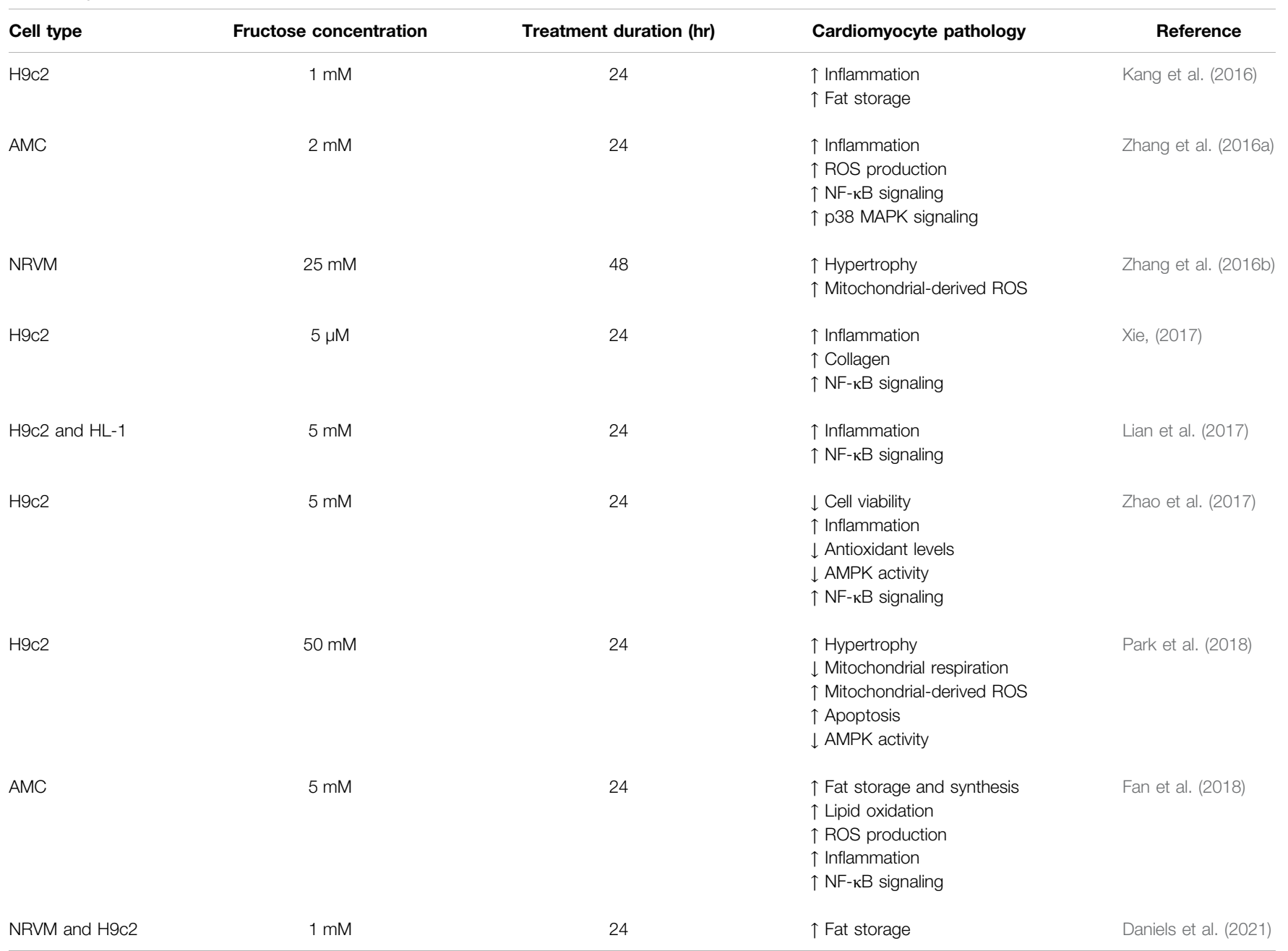

Rat cardiomyoblast cell line (H9c2), mouse atrial cell line (HL-1), adult mouse cardiomyocyte (AMC), neonatal rat ventricular myocyte (NRVM), reactive oxygen species (ROS), nuclear factor kappa-light-chain-enhancer of activated B cells (NF-kB), mitogen-activated protein kinase (MAPK), AMP-activated protein kinase (AMPK).

in faster glycation kinetics, production of Heyns products (a fructose homolog to Amadori products) and glycation of proteins and lipids (McPherson et al., 1988; Suárez et al., 1989; Schalkwijk et al., 2004; Takeuchi et al., 2010). Type 1 diabetic patients have 4fold higher circulating levels of fructose-derived AGEs relative to healthy controls (Takeuchi et al., 2010). Incubation of purified cardiac troponin- $\mathrm{C}$, the $\mathrm{Ca}^{2+}$ binding protein in the troponin complex which regulates cross-bridge cycling, with high fructose led to dramatically higher levels of AGE formation (carboxymethyllysine) and oxidation relative to high glucose conditions (Janssens et al., 2018). In non-cardiac cells, upregulation of the polyol pathway via sorbitol dehydrogenase overexpression increased fructose-derived AGEs (Takeuchi et al., 2010). Similarly, high fructose exposure increased fructosederived AGE formation in cultured human dendritic cells (Jaiswal et al., 2019). These studies suggest that elevated circulating fructose levels and intracellular fructose production (polyol pathway) in diabetes may have an increased capacity to produce AGEs.
Elevated intracellular fructose may also have the potential to modify cardiomyocyte function via O-GlcNAcylation posttranslational modification of proteins. In contrast to AGE formation, $O$-GlcNAcylation is a reversible modification of serine or threonine residues, mediated by an enzymatic process involving the hexosamine biosynthesis pathway. The glycolytic intermediate, fructose-6-phosphate, provides a substrate for UDP-GlcNAc, the precursor for $O$-GlcNAcylation. O-GlcNAcylation has been identified as an important mediator of cardiac pathologies (McLarty et al., 2013; Marsh et al., 2014) that may play a role in the development of diabetic cardiomyopathy (Hu et al., 2005; Erickson et al., 2013; Prakoso et al., 2021). Elevated levels of O-GlcNAcylation have been observed in left ventricular tissues of fructose-fed rodents (Hecker et al., 2012), but the direct involvement of fructose in the hexosamine biosynthesis pathway and O-GlcNAcylation in the heart has not been investigated. Evidence from non-cardiac cell lines indicates that fructose can promote $O$-GlcNAcylation, as increased UDP-O-GlcNAc levels are observed in HepG2 (liver) 
cells exposed to fructose for $24 \mathrm{~h}$ (Hirahatake et al., 2011). Together these studies suggest that fructose-derived $O$-GlcNAcylation is a potentially important signaling route.

\section{Fructose-Induced Cardiomyocyte Inflammation, Cell Death, and Pathological Growth}

Emerging evidence suggests that activation of inflammatory pathways is an important mediator of cardiac pathology in diabetes (Frati et al., 2017). In vitro studies have shown that cardiomyocyte fructose exposure upregulates the expression of pro-inflammatory cytokines, a hallmark of cellular stress (Kang et al., 2016; Zhang et al., 2016a; Lian et al., 2017; Xie, 2017; Zhao et al., 2017; Fan et al., 2018). Increased cytokine expression in this context is coupled to upregulated pro-inflammatory nuclear factor- $\kappa \mathrm{B}(\mathrm{NF}-\kappa \mathrm{B})$ and mitogen-activated protein kinases (MAPKs) pathways (Table 1), partly via interleukin-1 receptor-associated kinase 4/1 (IRAK4/1) and nucleotidebinding domain (NOD)-like receptor protein 4 (NLRP4) (Zhang et al., 2016a; Kang et al., 2016; Lian et al., 2017; Xie, 2017; Zhao et al., 2017). Fructose-induced cardiac inflammation may also be associated with the production of reactive oxygen species (ROS), and fructose exposure has been shown to increase ROS and suppress antioxidant defense systems in cultured cardiomyocytes (Zhao et al., 2017; Fan et al., 2018; Park et al., 2018). Upregulated cardiac inflammation and ROS production may underlie fructose-induced increase in apoptotic indices in primary mouse cardiomyocytes (Fan et al., 2018) and H9c2 cardiomyoblasts (Zhao et al., 2017; Park et al., 2018), coincident with decreased cell viability (Zhao et al., 2017). Interestingly, a glucagon-like peptide-1 (GLP-1) agonist has been shown to ameliorate fructose-induced cell death in cultured neuronal cells (Khalilnezhad and Taskiran, 2019), but to date this has not been investigated in cardiomyocytes. GLP-1 agonists are an emerging class of T2D therapies and the modes of action involve signaling, metabolic and functional pathways (Rowlands et al., 2018). Investigation into a role for GLP-1 signaling in regulating fructose cardiomyocyte toxicity would be informative. Inflammatory cytokines have also been linked to the development of cardiac hypertrophy and fructose exposure in vitro increases the production of tumor necrosis factor (TNF)- $\alpha$, interleukin (IL)-1 $\beta$, IL-6 and transforming growth factor (TGF)- $\beta 1$ in cardiomyocytes (Zhang et al., 2016a; Kang et al., 2016; Lian et al., 2017; Xie, 2017; Zhao et al., 2017; Fan et al., 2018). These cytokines are suggested to influence cardiac growth through upregulation of growth-related signaling pathways such as MAPK-JNK and CaMKII-STAT3 (Thaik et al., 1995; Condorelli et al., 2002; Matsumoto-Ida et al., 2006; Zhang et al., 2016a; Zhao

\section{REFERENCES}

Anderson, E. J., Kypson, A. P., Rodriguez, E., Anderson, C. A., Lehr, E. J., and Neufer, P. D. (2009). Substrate-specific Derangements in Mitochondrial Metabolism and Redox Balance in the Atrium of the Type 2 Diabetic Human Heart. J. Am. Coll. Cardiol. 54, 1891-1898. doi:10.1016/j.jacc.2009.07.031 et al., 2016). In vitro, high fructose exposure (25-50 mM) increased cell size in $\mathrm{H} 9 \mathrm{c} 2$ cardiomyoblasts and neonatal rat ventricular myocytes (Zhang et al., 2016b; Park et al., 2018), linked with increased expression of hypertrophic markers (Park et al., 2018). Thus it is evident that fructose exposure in vitro promotes cardiomyocyte inflammation, cell death and pathological growth.

\section{CONCLUSION AND FUTURE PERSPECTIVES}

Emerging evidence suggests that elevated fructose exposure and upregulated fructose metabolism may have significant impact on cardiomyocytes. Recent findings that diabetic patients exhibit elevated cardiac fructose and sorbitol content provide an important platform for ongoing work to understand the consequences of elevated fructose in the heart. In vitro studies have demonstrated that cardiomyocyte fructose exposure is linked with metabolic disturbance, lipid accumulation, inflammation, apoptosis and pathological growth. Further, intracellular fructose has the potential for post-translational modification of proteins, which may constitute an important mechanistic pathway for fructose-mediated disturbances in cardiomyocyte signaling and function. Significant knowledge gaps remain, and future investigations translating the key in vitro findings to an in vivo context are now warranted. Intervention studies targeting fructose-related pathways in the heart (e.g., fructose transport, fructose metabolism, the polyol pathway, fructose-derived AGEs) will provide important information on the potential role of fructose in mediating cardiac pathology. Fructose metabolism may prove to be an effective therapeutic target to mitigate the metabolic disturbances evident in diabetic heart disease, and understanding the role of fructose in the heart, in health and in disease states, is an important priority.

\section{AUTHOR CONTRIBUTIONS}

MA, LD, and KM drafted the manuscript. All authors edited, revised, and approved the final version of this review.

\section{FUNDING}

This work is supported by the Health Research Council of New Zealand (16/300) and the Maurice Wilkins Centre for Molecular Biodiscovery.

Axelsen, L. N., Lademann, J. B., Petersen, J. S., Holstein-Rathlou, N.-H., Ploug, T., Prats, C., et al. (2010). Cardiac and Metabolic Changes in Long-Term High Fructose-Fat Fed Rats with Severe Obesity and Extensive Intramyocardial Lipid Accumulation. Am. J. Physiology-Regulatory, Integr. Comp. Physiol. 298, R1560-R1570. doi:10.1152/ajpregu.00392.2009

Berg, T. J., Snorgaard, O., Faber, J., Torjesen, P. A., Hildebrandt, P., Mehlsen, J., et al. (1999). Serum Levels of Advanced Glycation End Products Are Associated 
with Left Ventricular Diastolic Function in Patients with Type 1 Diabetes. Diabetes Care 22, 1186-1190. doi:10.2337/diacare.22.7.1186

Bidasee, K. R., Nallani, K., Yu, Y., Cocklin, R. R., Zhang, Y., Wang, M., et al. (2003). Chronic Diabetes Increases Advanced Glycation End Products on Cardiac Ryanodine Receptors/calcium-Release Channels. Diabetes 52, 1825-1836. doi:10.2337/diabetes.52.7.1825

Bidasee, K. R., Zhang, Y., Shao, C. H., Wang, M., Patel, K. P., Dincer, U. D., et al. (2004). Diabetes Increases Formation of Advanced Glycation End Products on Sarco(endo)plasmic Reticulum Ca2+-ATPase. Diabetes 53, 463-473. doi:10.2337/diabetes.53.2.463

Bodiga, V. L., Eda, S. R., and Bodiga, S. (2014). Advanced Glycation End Products: Role in Pathology of Diabetic Cardiomyopathy. Heart Fail. Rev. 19, 49-63. doi:10.1007/s10741-013-9374-y

Boudina, S., Sena, S., O'Neill, B. T., Tathireddy, P., Young, M. E., and Abel, E. D. (2005). Reduced Mitochondrial Oxidative Capacity and Increased Mitochondrial Uncoupling Impair Myocardial Energetics in Obesity. Circulation 112, 2686-2695. doi:10.1161/CIRCULATIONAHA. 105.554360

Burant, C. F., Takeda, J., Brot-Laroche, E., Bell, G. I., and Davidson, N. O. (1992). Fructose Transporter in Human Spermatozoa and Small Intestine Is GLUT5. J. Biol. Chem. 267, 14523-14526. doi:10.1016/s0021-9258(18)42067-4

Cho, N. H., Shaw, J. E., Karuranga, S., Huang, Y., da Rocha Fernandes, J. D., Ohlrogge, A. W., et al. (2018). IDF Diabetes Atlas: Global Estimates of Diabetes Prevalence for 2017 and Projections for 2045. Diabetes Res. Clin. Pract. 138, 271-281. doi:10.1016/j.diabres.2018.02.023

Cho, S.-J., Roman, G., Yeboah, F., and Konishi, Y. (2007). The Road to Advanced Glycation End Products: a Mechanistic Perspective. Curr. Med. Chem. 14, 1653-1671. doi:10.2174/092986707780830989

Condorelli, G., Morisco, C., Latronico, M. V. G., Claudio, P. P., Dent, P., Tsichlis, P., et al. (2002). TNF- $\alpha$ Signal Transduction in Rat Neonatal Cardiac Myocytes: Definition of Pathways Generating from the TNF- $\alpha$ Receptor. FASEB j. 16, 1732-1737. doi:10.1096/fj.02-0419com

Daniels, L. J., Annandale, M., Koutsifeli, P., Li, X., Bussey, C. T., van Hout, I., et al. (2021). Elevated Myocardial Fructose and Sorbitol Levels Are Associated with Diastolic Dysfunction in Diabetic Patients, and Cardiomyocyte Lipid Inclusions In Vitro. Nutr. Diabetes 11, 8. doi:10.1038/s41387-021-00150-7

David, E. S., Cingari, D. S., and Ferraris, R. P. (1995). Dietary Induction of Intestinal Fructose Absorption in Weaning Rats. Pediatr. Res. 37, 777-782. doi:10.1203/00006450-199506000-00017

Delbridge, L. M. D., Benson, V. L., Ritchie, R. H., and Mellor, K. M. (2016). Diabetic Cardiomyopathy: The Case for a Role of Fructose in Disease Etiology. Diabetes 65, 3521-3528. doi:10.2337/db16-0682

Desrois, M., Sidell, R. J., Gauguier, D., King, L. M., Radda, G. K., and Clarke, K. (2004). Initial Steps of Insulin Signaling and Glucose Transport Are Defective in the Type 2 Diabetic Rat Heart. Cardiovasc. Res. 61, 288-296. doi:10.1016/ j.cardiores.2003.11.021

Diamant, M., Lamb, H. J., Groeneveld, Y., Endert, E. L., Smit, J. W. A., Bax, J. J., et al. (2003). Diastolic Dysfunction Is Associatedwith Altered Myocardial Metabolism Inasymptomatic Normotensive Patientswith Well-Controlled Type 2 Diabetes Mellitus. J. Am. Coll. Cardiol. 42, 328-335. doi:10.1016/ s0735-1097(03)00625-9

DiNicolantonio, J. J., O’Keefe, J. H., and Lucan, S. C. (2015). Added Fructose. Mayo Clinic Proc. 90, 372-381. doi:10.1016/j.mayocp.2014.12.019

Douard, V., and Ferraris, R. P. (2008). Regulation of the Fructose Transporter GLUT5 in Health and Disease. Am. J. Physiology-Endocrinology Metab. 295, E227-E237. doi:10.1152/ajpendo.90245.2008

Drozdowski, L., and Thomson, A. B. R. (2006). Intestinal Sugar Transport. World J. Gastroenterol. 12, 1657-1670. doi:10.3748/wjg.v12.i11.1657

Erickson, J. R., Pereira, L., Wang, L., Han, G., Ferguson, A., Dao, K., et al. (2013). Diabetic Hyperglycaemia Activates CaMKII and Arrhythmias by O-Linked Glycosylation. Nature 502, 372-376. doi:10.1038/nature12537

Faeh, D., Minehira, K., Schwarz, J.-M., Periasamy, R., Park, S., Tappy, L., et al. (2005). Effect of Fructose Overfeeding and Fish Oil Administration on Hepatic De Novo Lipogenesis and Insulin Sensitivity in Healthy Men. Diabetes 54, 1907-1913. doi:10.2337/diabetes.54.7.1907

Fan, X.-D., Wan, L.-L., Duan, M., and Lu, S. (2018). HDAC11 Deletion Reduces Fructose-Induced Cardiac Dyslipidemia, Apoptosis and Inflammation by
Attenuating Oxidative Stress Injury. Biochem. Biophysical Res. Commun. 503, 444-451. doi:10.1016/j.bbrc.2018.04.090

Ford, E. S., Giles, W. H., and Dietz, W. H. (2002). Prevalence of the Metabolic Syndrome Among US Adults. JAMA 287, 356-359. doi:10.1001/jama.287.3.356

Francey, C., Cros, J., Rosset, R., Crézé, C., Rey, V., Stefanoni, N., et al. (2019). The Extra-splanchnic Fructose Escape after Ingestion of a Fructose-Glucose Drink: An Exploratory Study in Healthy Humans Using a Dual Fructose Isotope Method. Clin. Nutr. ESPEN 29, 125-132. doi:10.1016/j.clnesp.2018.11.008

Frati, G., Schirone, L., Chimenti, I., Yee, D., Biondi-Zoccai, G., Volpe, M., et al. (2017). An Overview of the Inflammatory Signalling Mechanisms in the Myocardium Underlying the Development of Diabetic Cardiomyopathy. Cardiovasc. Res. 113, 378-388. doi:10.1093/cvr/cvx011

Gul, A., Rahman, M. A., and Hasnain, S. N. (2009). Influence of Fructose Concentration on Myocardial Infarction in Senile Diabetic and Non-diabetic Patients. Exp. Clin. Endocrinol. Diabetes 117, 605-609. doi:10.1055/s-00291202793

Hecker, P. A., Mapanga, R. F., Kimar, C. P., Ribeiro, R. F., Brown, B. H., O’Connell, K. A., et al. (2012). Effects of Glucose-6-Phosphate Dehydrogenase Deficiency on the Metabolic and Cardiac Responses to Obesogenic or High-Fructose Diets. Am. J. Physiology-Endocrinology Metab. 303, E959-E972. doi:10.1152/ ajpendo.00202.2012

Hirahatake, K. M., Meissen, J. K., Fiehn, O., and Adams, S. H. (2011). Comparative Effects of Fructose and Glucose on Lipogenic Gene Expression and Intermediary Metabolism in HepG2 Liver Cells. PLoS One 6, e26583. doi:10.1371/journal.pone.0026583

Hu, Y., Belke, D., Suarez, J., Swanson, E., Clark, R., Hoshijima, M., et al. (2005). Adenovirus-Mediated Overexpression of $\mathrm{O}$-GlcNAcase Improves Contractile Function in the Diabetic Heart. Circ. Res. 96, 1006-1013. doi:10.1161/ 01.RES.0000165478.06813.58

Hui, H., Huang, D., McArthur, D., Nissen, N., Boros, L. G., and Heaney, A. P. (2009). Direct Spectrophotometric Determination of Serum Fructose in Pancreatic Cancer Patients. Pancreas 38, 706-712. doi:10.1097/ MPA.0b013e3181a7c6e5

Huynh, K., Bernardo, B. C., McMullen, J. R., and Ritchie, R. H. (2014). Diabetic Cardiomyopathy: Mechanisms and New Treatment Strategies Targeting Antioxidant Signaling Pathways. Pharmacol. Ther. 142, 375-415. doi:10.1016/j.pharmthera.2014.01.003

Iwata, K., Nishinaka, T., Matsuno, K., Kakehi, T., Katsuyama, M., Ibi, M., et al. (2007). The Activity of Aldose Reductase Is Elevated in Diabetic Mouse Heart. J. Pharmacol. Sci. 103, 408-416. doi:10.1254/jphs.fp0070136

Jaiswal, N., Agrawal, S., and Agrawal, A. (2019). High Fructose-induced Metabolic Changes Enhance Inflammation in Human Dendritic Cells. Clin. Exp. Immunol. 197, 237-249. doi:10.1111/cei.13299

Jang, C., Hui, S., Lu, W., Cowan, A. J., Morscher, R. J., Lee, G., et al. (2018). The Small Intestine Converts Dietary Fructose into Glucose and Organic Acids. $\mathrm{Cel}$ Metab. 27, 351-361. doi:10.1016/j.cmet.2017.12.016

Janssens, J. V., Ma, B., Brimble, M. A., Van Eyk, J. E., Delbridge, L. M. D., and Mellor, K. M. (2018). Cardiac Troponins May Be Irreversibly Modified by Glycation: Novel Potential Mechanisms of Cardiac Performance Modulation. Sci. Rep. 8, 16084. doi:10.1038/s41598-018-33886-x

Jiang, L., David, E. S., Espina, N., and Ferraris, R. P. (2001). GLUT-5 Expression in Neonatal Rats: Crypt-Villus Location and Age-dependent Regulation. Am. J. Physiology-Gastrointestinal Liver Physiol. 281, G666-G674. doi:10.1152/ ajpgi.2001.281.3.G666

Johnson, B. F., Nesto, R. W., Pfeifer, M. A., Slater, W. R., Vinik, A. I., Chyun, D. A. et al. (2004). Cardiac Abnormalities in Diabetic Patients with Neuropathy: Effects of Aldose Reductase Inhibitor Administration. Diabetes Care 27, 448-454. doi:10.2337/diacare.27.2.448

Johnson, R. J., Nakagawa, T., Sanchez-Lozada, L. G., Shafiu, M., Sundaram, S., Le, M., et al. (2013). Sugar, Uric Acid, and the Etiology of Diabetes and Obesity. Diabetes 62, 3307-3315. doi:10.2337/db12-1814

Kang, L.-L., Zhang, D.-M., Ma, C.-H., Zhang, J.-H., Jia, K.-K., Liu, J.-H., et al. (2016). Cinnamaldehyde and Allopurinol Reduce Fructose-Induced Cardiac Inflammation and Fibrosis by Attenuating CD36-Mediated TLR4/6-Irak4/1 Signaling to Suppress NLRP3 Inflammasome Activation. Sci. Rep. 6, 27460. doi:10.1038/srep27460

Kashiwagi, A., Obata, T., Suzaki, M., Takagi, Y., Kida, Y., Ogawa, T., et al. (1992). Increase in Cardiac Muscle Fructose Content in Streptozotocin- 
Induced Diabetic Rats. Metabolism 41, 1041-1046. doi:10.1016/00260495(92)90283-g

Kawasaki, T., Akanuma, H., and Yamanouchi, T. (2002). Increased Fructose Concentrations in Blood and Urine in Patients with Diabetes. Diabetes Care 25, 353-357. doi:10.2337/diacare.25.2.353

Kayano, T., Burant, C. F., Fukumoto, H., Gould, G. W., Fan, Y. S., Eddy, R. L., et al. (1990). Human Facilitative Glucose Transporters. Isolation, Functional Characterization, and Gene Localization of cDNAs Encoding an Isoform (GLUT5) Expressed in Small Intestine, Kidney, Muscle, and Adipose Tissue and an Unusual Glucose Transporter Pseudogene-like Sequence (GLUT6). J. Biol. Chem. 265, 13276-13282. doi:10.1016/s00219258(19)38295-x

Khalilnezhad, A., and Taskiran, D. (2019). The Investigation of Protective Effects of Glucagon-like Peptide-1 (GLP-1) Analogue Exenatide against Glucose and Fructose-Induced Neurotoxicity. Int. J. Neurosci. 129, 481-491. doi:10.1080/ 00207454.2018.1543671

Khitan, Z., and Kim, D. H. (2013). Fructose: a Key Factor in the Development of Metabolic Syndrome and Hypertension. J. Nutr. Metab. 2013, 1-12. doi:10.1155/2013/682673

Kolwicz, S. C., Purohit, S., and Tian, R. (2013). Cardiac Metabolism and its Interactions With Contraction, Growth, and Survival of Cardiomyocytes. Circ. Res. 113, 603-616. doi:10.1161/CIRCRESAHA.113.302095

Kruszynska, Y. T., Meyer-Alber, A., Wollen, N., and McIntyre, N. (1993). Energy Expenditure and Substrate Metabolism after Oral Fructose in Patients with Cirrhosis. J. Hepatol. 19, 241-251. doi:10.1016/s0168-8278(05)80578-1

Lal, S., Randall, W. C., Taylor, A. H., Kappler, F., Walker, M., Brown, T. R., et al. (1997). Fructose-3-phosphate Production and Polyol Pathway Metabolism in Diabetic Rat Hearts. Metabolism 46, 1333-1338. doi:10.1016/s0026-0495(97) 90240-7

Lalowski, M. M., Björk, S., Finckenberg, P., Soliymani, R., Tarkia, M., Calza, G., et al. (2018). Characterizing the Key Metabolic Pathways of the Neonatal Mouse Heart Using a Quantitative Combinatorial Omics Approach. Front. Physiol. 9, 365. doi:10.3389/fphys.2018.00365

Le, M. T., Frye, R. F., Rivard, C. J., Cheng, J., McFann, K. K., Segal, M. S., et al. (2012). Effects of High-Fructose Corn Syrup and Sucrose on the Pharmacokinetics of Fructose and Acute Metabolic and Hemodynamic Responses in Healthy Subjects. Metabolism 61, 641-651. doi:10.1016/ j.metabol.2011.09.013

Leturque, A., Brot-Laroche, E., Le Gall, M., Stolarczyk, E., and Tobin, V. (2005). The Role of GLUT2 in Dietary Sugar Handling. J. Physiol. Biochem. 61, 529-537. doi:10.1007/BF03168378

Li, Q., Hwang, Y. C., Ananthakrishnan, R., Oates, P. J., Guberski, D., and Ramasamy, R. (2008). Polyol Pathway and Modulation of IschemiaReperfusion Injury in Type 2 Diabetic BBZ Rat Hearts. Cardiovasc. Diabetol. 7, 33. doi:10.1186/1475-2840-7-33

Lian, Y.-G., Zhao, H.-Y., Wang, S.-J., Xu, Q.-L., and Xia, X.-J. (2017). NLRP4 Is an Essential Negative Regulator of Fructose-Induced Cardiac Injury In Vitro and In Vivo. Biomed. Pharmacother. 91, 590-601. doi:10.1016/ j.biopha.2017.04.120

Lind, M., Svensson, A.-M., Kosiborod, M., Gudbjörnsdottir, S., Pivodic, A., Wedel, H., et al. (2014). Glycemic Control and Excess Mortality in Type 1 Diabetes. N. Engl. J. Med. 371, 1972-1982. doi:10.1056/NEJMoa1408214

Low, W., Cornfield, T., Charlton, C., Tomlinson, J., and Hodson, L. (2018). Sex Differences in Hepatic De Novo Lipogenesis with Acute Fructose Feeding. Nutrients 10, 1263. doi:10.3390/nu10091263

Malik, V. S., Popkin, B. M., Bray, G. A., Després, J.-P., Willett, W. C., and Hu, F. B. (2010). Sugar-sweetened Beverages and Risk of Metabolic Syndrome and Type 2 Diabetes: a Meta-Analysis. Diabetes Care 33, 2477-2483. doi:10.2337/dc101079

Marsh, S. A., Collins, H. E., and Chatham, J. C. (2014). Protein O-GlcNAcylation and Cardiovascular (Patho)physiology. J. Biol. Chem. 289, 34449-34456. doi:10.1074/jbc.R114.585984

Matsumoto-Ida, M., Takimoto, Y., Aoyama, T., Akao, M., Takeda, T., and Kita, T. (2006). Activation of TGF- $\beta 1-T A K 1-P 38$ MAPK Pathway in Spared Cardiomyocytes Is Involved in Left Ventricular Remodeling after Myocardial Infarction in Rats. Am. J. Physiology-Heart Circulatory Physiol. 290, H709-H715. doi:10.1152/ajpheart.00186.2005
McLarty, J. L., Marsh, S. A., and Chatham, J. C. (2013). Post-translational Protein Modification by O-Linked N-Acetyl-Glucosamine: its Role in Mediating the Adverse Effects of Diabetes on the Heart. Life Sci. 92, 621-627. doi:10.1016/ j.lfs.2012.08.006

McPherson, J. D., Shilton, B. H., and Walton, D. J. (1988). Role of Fructose in Glycation and Cross-Linking of Proteins. Biochemistry 27, 1901-1907. doi:10.1021/bi00406a016

Mellor, K. M., Bell, J. R., Wendt, I. R., Davidoff, A. J., Ritchie, R. H., and Delbridge, L. M. D. (2011a). Fructose Modulates Cardiomyocyte Excitation-Contraction Coupling and Ca2+ Handling In Vitro. PLoS One 6, e25204. doi:10.1371/ journal.pone.0025204

Mellor, K. M., Bell, J. R., Young, M. J., Ritchie, R. H., and Delbridge, L. M. D. (2011b). Myocardial Autophagy Activation and Suppressed Survival Signaling Is Associated with Insulin Resistance in Fructose-Fed Mice. J. Mol. Cell Cardiol. 50, 1035-1043. doi:10.1016/j.yjmcc.2011.03.002

Mellor, K. M., Ritchie, R. H., Davidoff, A. J., and Delbridge, L. M. D. (2010a). Elevated Dietary Sugar and the Heart: Experimental Models and Myocardial Remodeling. Can. J. Physiol. Pharmacol. 88, 525-540. doi:10.1139/y10-005

Mellor, K. M., Wendt, I. R., Ritchie, R. H., and Delbridge, L. M. D. (2012). Fructose Diet Treatment in Mice Induces Fundamental Disturbance of Cardiomyocyte Ca2+ Handling and Myofilament Responsiveness. Am. J. Physiology-Heart Circulatory Physiol. 302, H964-H972. doi:10.1152/ajpheart.00797.2011

Mellor, K., Ritchie, R. H., Meredith, G., Woodman, O. L., Morris, M. J., and Delbridge, L. M. D. (2010b). High-fructose Diet Elevates Myocardial Superoxide Generation in Mice in the Absence of Cardiac Hypertrophy. Nutrition 26, 842-848. doi:10.1016/j.nut.2009.08.017

Mirtschink, P., and Krek, W. (2016). Hypoxia-driven Glycolytic and Fructolytic Metabolic Programs: Pivotal to Hypertrophic Heart Disease. Biochim. Biophys. Acta (BBA) - Mol. Cel Res. 1863, 1822-1828. doi:10.1016/j.bbamcr.2016.02.011

Mirtschink, P., Krishnan, J., Grimm, F., Sarre, A., Hörl, M., Kayikci, M., et al. (2015). HIF-driven SF3B1 Induces KHK-C to Enforce Fructolysis and Heart Disease. Nature 522, 444-449. doi:10.1038/nature14508

Nichols, G. A., Hillier, T. A., Erbey, J. R., and Brown, J. B. (2001). Congestive Heart Failure in Type 2 Diabetes: Prevalence, Incidence, and Risk Factors. Diabetes Care 24, 1614-1619. doi:10.2337/diacare.24.9.1614

Papadaki, M., Holewinski, R. J., Previs, S. B., Martin, T. G., Stachowski, M. J., Li, A., et al. (2018). Diabetes with Heart Failure Increases Methylglyoxal Modifications in the Sarcomere, Which Inhibit Function. JCI Insight 3. e121264. doi:10.1172/ jci.insight. 121264

Park, J. H., Ku, H. J., Kim, J. K., Park, J.-W., and Lee, J. H. (2018). Amelioration of High Fructose-Induced Cardiac Hypertrophy by Naringin. Sci. Rep. 8, 9464. doi:10.1038/s41598-018-27788-1

Patel, C., Douard, V., Yu, S., Gao, N., and Ferraris, R. P. (2015). Transport, Metabolism, and Endosomal Trafficking-dependent Regulation of Intestinal Fructose Absorption. FASEB j. 29, 4046-4058. doi:10.1096/fj.15-272195

Pereira, R., Botezelli, J., da Cruz Rodrigues, K., Mekary, R., Cintra, D., Pauli, J., et al. (2017). Fructose Consumption in the Development of Obesity and the Effects of Different Protocols of Physical Exercise on the Hepatic Metabolism. Nutrients 9, 405. doi:10.3390/nu9040405

Pham, T., Loiselle, D., Power, A., and Hickey, A. J. R. (2014). Mitochondrial Inefficiencies and Anoxic ATP Hydrolysis Capacities in Diabetic Rat Heart. Am. J. Physiology-Cell Physiol. 307, C499-C507. doi:10.1152/ajpcell.00006.2014

Prakoso, D., Lim, S. Y., Erickson, J. R., Wallace, R. S., Lees, J. G., Tate, M., et al. (2021). Fine-tuning the Cardiac O-GlcNAcylation Regulatory Enzymes Governs the Functional and Structural Phenotype of the Diabetic Heart. Cardiovasc. Res. [Epub ahead of print]. doi:10.1093/cvr/cvab043

Regan, T. J., Lyons, M. M., Ahmed, S. S., Levinson, G. E., Oldewurtel, H. A., Ahmad, M. R., et al. (1977). Evidence for Cardiomyopathy in Familial Diabetes Mellitus. J. Clin. Invest. 60, 885-899. doi:10.1172/JCI108843

Ritchie, R. H., and Abel, E. D. (2020). Basic Mechanisms of Diabetic Heart Disease. Circ. Res. 126, 1501-1525. doi:10.1161/CIRCRESAHA.120.315913

Rogers, S., Chandler, J. D., Clarke, A. L., Petrou, S., and Best, J. D. (2003). Glucose Transporter GLUT12-Functional Characterization in Xenopus laevis Oocytes. Biochem. Biophysical Res. Commun. 308, 422-426. doi:10.1016/s0006-291x(03) 01417-7

Rogers, S., Macheda, M. L., Docherty, S. E., Carty, M. D., Henderson, M. A., Soeller, W. C., et al. (2002). Identification of a Novel Glucose Transporter-like Protein- 
GLUT-12. Am. J. Physiol. Endocrinol. Metab. 282, E733-E738. doi:10.1152/ ajpendo.2002.282.3.E733

Rowlands, J., Heng, J., Newsholme, P., and Carlessi, R. (2018). Pleiotropic Effects of GLP-1 and Analogs on Cell Signaling, Metabolism, and Function. Front. Endocrinol. 9, 672. doi:10.3389/fendo.2018.00672

Rumessen, J. J. (1992). Fructose and Related Food Carbohydrates: Sources, Intake, Absorption, and Clinical Implications. Scand. J. Gastroenterol. 27, 819-828. doi:10.3109/00365529209000148

Saponaro, C., Gaggini, M., Carli, F., and Gastaldelli, A. (2015). The Subtle Balance between Lipolysis and Lipogenesis: A Critical Point in Metabolic Homeostasis. Nutrients 7, 9453-9474. doi:10.3390/nu7115475

Schalkwijk, C. G., Stehouwer, C. D. A., and van Hinsbergh, V. W. M. (2004). Fructose-mediated Non-enzymatic Glycation: Sweet Coupling or Bad Modification. Diabetes Metab. Res. Rev. 20, 369-382. doi:10.1002/dmrr.488

Scheepers, A., Schmidt, S., Manolescu, A., Cheeseman, C. I., Bell, A., Zahn, C., et al. (2005). Characterization of the humanSLC2A11(GLUT11) Gene: Alternative Promoter Usage, Function, Expression, and Subcellular Distribution of Three Isoforms, and Lack of Mouse Orthologue. Mol. Membr. Biol. 22, 339-351. doi:10.1080/09687860500166143

Sharavana, G., Joseph, G. S., and Baskaran, V. (2017). Lutein Attenuates Oxidative Stress Markers and Ameliorates Glucose Homeostasis through Polyol Pathway in Heart and Kidney of STZ-Induced Hyperglycemic Rat Model. Eur. J. Nutr. 56, 2475-2485. doi:10.1007/s00394-016-1283-0

Stanhope, K. L., Schwarz, J. M., Keim, N. L., Griffen, S. C., Bremer, A. A., Graham, J. L., et al. (2009). Consuming Fructose-Sweetened, Not Glucose-Sweetened, Beverages Increases Visceral Adiposity and Lipids and Decreases Insulin Sensitivity in Overweight/obese Humans. J. Clin. Invest. 119, 1322-1334. doi:10.1172/JCI37385

Stanhope, K. L. (2016). Sugar Consumption, Metabolic Disease and Obesity: The State of the Controversy. Crit. Rev. Clin. Lab. Sci. 53, 52-67. doi:10.3109/ 10408363.2015.1084990

Suárez, G., Rajaram, R., Oronsky, A. L., and Gawinowicz, M. A. (1989). Nonenzymatic Glycation of Bovine Serum Albumin by Fructose (Fructation). J. Biol. Chem. 264, 3674-3679. doi:10.1016/s0021-9258(19)84904-9

Sun, S. Z., and Empie, M. W. (2012). Fructose Metabolism in Humans - what Isotopic Tracer Studies Tell Us. Nutr. Metab. 9, 89. doi:10.1186/1743-7075-9-89

Sveen, K. A., Nerdrum, T., Hanssen, K. F., Brekke, M., Torjesen, P. A., Strauch, C. M., et al. (2014). Impaired Left Ventricular Function and Myocardial Blood Flow reserve in Patients with Long-Term Type 1 Diabetes and No Significant Coronary Artery Disease: Associations with Protein Glycation. Diabetes Vasc. Dis. Res. 11, 84-91. doi:10.1177/1479164113518805

Szűcs, G., Sója, A., Péter, M., Sárközy, M., Bruszel, B., Siska, A., et al. (2019). Prediabetes Induced by Fructose-Enriched Diet Influences Cardiac Lipidome and Proteome and Leads to Deterioration of Cardiac Function Prior to the Development of Excessive Oxidative Stress and Cell Damage. Oxid Med. Cel Longev 2019, 3218275. doi:10.1155/2019/3218275

Takeuchi, M., Iwaki, M., Takino, J.-i., Shirai, H., Kawakami, M., Bucala, R., et al. (2010). Immunological Detection of Fructose-Derived Advanced Glycation End-Products. Lab. Invest. 90, 1117-1127. doi:10.1038/labinvest.2010.62

Tancredi, M., Rosengren, A., Svensson, A.-M., Kosiborod, M., Pivodic, A., Gudbjörnsdottir, S., et al. (2015). Excess Mortality Among Persons with Type 2 Diabetes. N. Engl. J. Med. 373, 1720-1732. doi:10.1056/NEJMoa1504347
Tang, W. H., Cheng, W. T., Kravtsov, G. M., Tong, X. Y., Hou, X. Y., Chung, S K., et al. (2010). Cardiac Contractile Dysfunction during Acute Hyperglycemia Due to Impairment of SERCA by Polyol PathwayMediated Oxidative Stress. Am. J. Physiology-Cell Physiol. 299, C643-C653. doi:10.1152/ajpcell.00137.2010

Tang, W. H., Martin, K. A., and Hwa, J. (2012). Aldose Reductase, Oxidative Stress, and Diabetic Mellitus. Front. Pharmacol. 3, 87. doi:10.3389/ fphar.2012.00087

Thaik, C. M., Calderone, A., Takahashi, N., and Colucci, W. S. (1995). Interleukin1 Beta Modulates the Growth and Phenotype of Neonatal Rat Cardiac Myocytes. J. Clin. Invest. 96, 1093-1099. doi:10.1172/JCI118095

Tran, C., Jacot-Descombes, D., Lecoultre, V., Fielding, B. A., Carrel, G., Lê, K.-A., et al. (2010). Sex Differences in Lipid and Glucose Kinetics after Ingestion of an Acute Oral Fructose Load. Br. J. Nutr. 104, 1139-1147. doi:10.1017/ S000711451000190X

van den Brom, C. E., Huisman, M. C., Vlasblom, R., Boontje, N. M., Duijst, S., Lubberink, M., et al. (2009). Altered Myocardial Substrate Metabolism Is Associated with Myocardial Dysfunction in Early Diabetic Cardiomyopathy in Rats: Studies Using Positron Emission Tomography. Cardiovasc. Diabetol. 8, 39. doi:10.1186/1475-2840-8-39

Xie, X.-W. (2017). Liquiritigenin Attenuates Cardiac Injury Induced by High Fructose-Feeding through Fibrosis and Inflammation Suppression. Biomed. Pharmacother. 86, 694-704. doi:10.1016/j.biopha.2016.12.066

Zhang, Y. B., Meng, Y. H., Chang, S., Zhang, R. Y., and Shi, C. (2016b). High Fructose Causes Cardiac Hypertrophy via Mitochondrial Signaling Pathway. Am. J. Transl Res. 8, 4869-4880.

Zhang, Y., Zhang, L., Zhang, Y., Xu, J.-J., Sun, L.-L., and Li, S.-Z. (2016a). The Protective Role of Liquiritin in High Fructose-Induced Myocardial Fibrosis via Inhibiting NF- $\mathrm{kB}$ and MAPK Signaling Pathway. Biomed. Pharmacother. 84, 1337-1349. doi:10.1016/j.biopha.2016.10.036

Zhao, C., Zhang, Y., Liu, H., Li, P., Zhang, H., and Cheng, G. (2017). Fortunellin Protects against High Fructose-Induced Diabetic Heart Injury in Mice by Suppressing Inflammation and Oxidative Stress via AMPK/Nrf-2 Pathway Regulation. Biochem. Biophysical Res. Commun. 490, 552-559. doi:10.1016/ j.bbrc.2017.06.076

Zhao, L., Cheng, G., Jin, R., Afzal, M. R., Samanta, A., Xuan, Y.-T., et al. (2016). Deletion of Interleukin-6 Attenuates Pressure Overload-Induced Left Ventricular Hypertrophy and Dysfunction. Circ. Res. 118, 1918-1929. doi:10.1161/CIRCRESAHA.116.308688

Conflict of Interest: The authors declare that the research was conducted in the absence of any commercial or financial relationships that could be construed as a potential conflict of interest.

Copyright (c) 2021 Annandale, Daniels, Li, Neale, Chau, Ambalawanar, James, Koutsifeli, Delbridge and Mellor. This is an open-access article distributed under the terms of the Creative Commons Attribution License (CC BY). The use, distribution or reproduction in other forums is permitted, provided the original author(s) and the copyright owner(s) are credited and that the original publication in this journal is cited, in accordance with accepted academic practice. No use, distribution or reproduction is permitted which does not comply with these terms. 